upf. $\begin{array}{ll}\text { Universitat } \\ \text { Pompeu Fabra } \\ \text { Barcelona }\end{array} \quad \begin{aligned} & \text { Department } \\ & \text { of Economics and Business }\end{aligned}$

Economics Working Paper Series

Working Paper No. 1674

\title{
Underpromise and overdeliver? - Online product reviews and firm pricing
}

Simon Martin and Sandro Shelegia

October 2019 


\title{
Underpromise and Overdeliver? - \\ Online Product Reviews and Firm pricing
}

\author{
Simon Martin* Sandro Shelegia ${ }^{\dagger}$
}

October 24, 2019

\begin{abstract}
This paper presents a quality signaling model with consumer reviews. Reviews reflect true quality as well as consumers' expectations of quality, improving with the former and worsening with the latter. Expectation-based reviews give rise to a novel separating equilibrium with several interesting properties: (i) low quality types are deterred from charging high prices by disappointed consumers who may write bad reviews; (ii) high quality types are deterred from imitating low types and thus generating good reviews by low equilibrium prices set by the low types; (iii) prices charged by lowest quality types can be below marginal cost. The equilibrium price schedule is inversely related to the number of consumers who rely on product reviews. Hence higher reliance on reviews reduces prices. In contrast, more informative reviews may lead to lower as well as higher prices depending on how reviews are generated. These results extend to the duopoly model, where we show that prices are lower (higher) than under monopoly for prices above (below) marginal cost.
\end{abstract}

Keywords: quality signaling, consumer reviews, reputation

JEL Codes: C73, D82, D83, L14, L15

\footnotetext{
${ }^{*}$ Corresponding author. Düsseldorf Institute for Competition Economics (DICE), University of Düsseldorf. Email: simon.martin@dice.hhu.de. I thankfully acknowledge funding through Vienna Graduate School of Economics.

†Universitat Pompeu Fabra and Barcelona GSE. Email: sandro.shelegia@upf.edu. I acknowledge financial support from the Spanish Ministry of Economy and Competitiveness, through the Severo Ochoa Programme for Centres of Excellence in R\&D (SEV-2015-0563)
} 


\section{Introduction}

For many consumers it has become natural to gather information about products online prior to purchase, and reviews of previous consumers are an important part of the information available. There is growing empirical evidence on the relationship between consumer reviews, demand and revenue (Chevalier and Mayzlin, 2006, Anderson and Magruder, 2012, Luca, 2016, Jin et al., 2018). Therefore, it is increasingly the case that firms' pricing and marketing choices need to take into account how consumers write reviews. Chevalier and Mayzlin (2006) show that firms manipulate reviews by creating fake positive ones for themselves and fake negative ones for close competitors. However, beyond cheating, there are many ways in which firms may be able to influence reviews for their products or services. These practices are poorly understood theoretically. In particular, this is the first paper that comprehensively studies (i) why and how consumers write reviews, and (ii) how firms respond to review creation with their prices or marketing strategies.

We build a theoretical model that studies review creation and firms' response in terms of their pricing. We start with the premise that consumers write reviews not only to convey the true quality of a product, but also to convey how the product fares relative to their prior expectation (which itself may depend on the price payed, as well as other factors such as marketing). The incorporation of expectations in the process of information transmission between consumers is motivated by anecdotal evidence that expectations matter for reviews. At a glance, many "5-star" reviews on websites such as Amazon.com do not try to say that this is the best product one could have purchased for the need, but that it is very good for the price (and thus relative to the prior expectation), a consumer payed for it. Similar situations arise when an otherwise well-functioning product gets 1-star reviews not because it is bad, but simply because it falls short of very high 
expectations commanded by its high price. $^{1}$

A consumer who reads reviews may be able to separately infer true quality and reviewer's expectations from the text, but many consumers do not have time or desire to do so, and thus rely on average star count when searching for alternatives. It is thus compelling to try to understand how firms selling a good of uncertain quality would price their goods if initial consumers inform later consumers through reviews, and such reviews reflected both true quality as well as the anticipated one.

We model a monopolist who introduces an experience good of quality known to it, but not to the consumers. The monopolist's pricing choice influences the quality expectations of the consumers and hence introductory period demand, where consumers do not have any information apart from the price. If early period consumers buy, they create either a good or a bad review according to a stochastic review function, which depends both on the true quality observed, but importantly also on the expectations consumers had prior to purchase. $^{2}$ Naturally, the probability of a good review increases in true quality. However, the probability also decreases in the prior expectation of quality, reflecting the idea that consumers are less likely to be satisfied with the product the higher their expectations. Post-introductory period consumers observe the review, but not the introductory period prices, and update their beliefs about product quality accordingly.

We derive a (D1-criterion satisfying) separating equilibrium for introductory period prices which perfectly reveals true product quality to consumers in the period. In the post-introductory period, prices are the same for all qualities conditional on the realized

\footnotetext{
${ }^{1}$ While there is no direct empirical evidence on this phenomenon, it is very easy to find examples such as the following reviews for the Fire TV Stick from Amazon.com:

- "Great product, exceeded my expectations." (5 stars)

- "Easy set up, works better than I expecter [sic]" (5 stars)

- "Wonderful addition to my TV. Enjoying it even more than expected." (5 stars)

- "It did not meet my expectations." (1 star)

- "I was disappointed. Was just expecting more." (1 star)

${ }^{2}$ In the appendix, we describe a behavioral model in which consumers exert effort to operate a product, depending on expected quality. We then model reviewers as merely reporting their success operating the product. Since consumer's effort decreases in expected quality, higher expected quality makes a good outcome and thus a good review less likely.
} 
review, and simply equal to the expected quality given the review. ${ }^{3}$ Given that a good review commands a higher price, the presence of the second period creates endogenous incentives to obtain a good review in the introductory period.

The monopolist with quality $q$ faces a trade-off in the choice of the introductory period price: charging a higher price than that corresponding to quality $q$, increases introductory period profit but also leads to higher expectations. When the product inevitably falls short of these elevated expectations, early consumers are likely to respond with a bad review, which reduces post-introductory period profits. Interestingly, reviews in our model also alter profitability of deviations downward. When a high quality firm charges lower than its equilibrium price, while it suffers introductory period loss, by inducing lower expectations upon which it is bound to overdeliver, it also increases the probability of a good review and thus higher profits in the future. Our separating equilibrium guarantees that deviations in either direction are not profitable. Namely, the equilibrium pricing schedule is increasing in quality, starts low (possibly below marginal cost) and grows until the highest quality type charges price equal to her quality (and thus fully extracts consumer surplus). The price schedule depends inversely on the price premium for a good review, which in itself depends on how informative reviews are.

To gain more insight into the equilibrium, in section 4 we impose additional structure on the review creation function. The review function has two effects on equilibrium. First, it alters informativeness of equilibrium reviews. The more informative reviews are, the lower is the introductory period price schedule. Second, the review function determines how responsive reviews are to changes in quality expectations, with more responsiveness to expectations leading to lower prices. We provide two examples of the review generation function. Both examples share the same equilibrium informativeness of the review function, which is parametrized with $\alpha$. In the first example $\alpha$ also makes reviews more responsive do expectations, so then an increase in $\alpha$ leads to lower prices. In the second example $\alpha$ makes reviews less responsive to expectations, and then equilibrium prices are decreasing in $\alpha$ for low initial $\alpha$ and increasing otherwise. The last example corresponds to a situation where as $\alpha$ increases more consumers report true quality, and then prices may go up or down.

\footnotetext{
${ }^{3}$ The presence of post-introductory period pooling equilibrium does not mean that in this period purchases are not based on any information. Reviews are correlated with true quality, and so are the purchases, however, given a realized review level, true quality does not affect price.
} 
We also consider an extension to duopoly (similar analysis applies for oligopoly). We find that the structure of the separating equilibrium is fully preserved with competition. Prices are still decreasing with the number of post-introductory consumers, and are lower the more responsive reviews are to expected quality. We find that under duopoly prices tend to be lower, except when prices are below marginal cost even under monopoly. Intuitively, a monopolist appropriates all the benefit from good reviews in the second period. If the second period is sufficiently important (many consumers arrive later), then low quality types have to charge prices far below marginal cost in order to deter high types from imitation. This force is stronger then under duopoly and hence prices are lower.

\section{Literature review}

There is a vast literature on quality signaling through prices. Shapiro (1983) and Milgrom and Roberts (1986) consider optimal pricing in a world with repeat purchases, so in contrast to our model, no information is transmitted between consumers. The pessimistic case of Shapiro (1983) where consumers initially underestimate true product quality describes a trade-off similar to ours: increasing early period prices results in less favorable beliefs about quality in the future. While in Shapiro's model this follows from lower demand in early periods and hence a smaller fraction of the population learning about true quality, in our model higher expectations and hence worse reviews drive future beliefs. However, Shapiro's optimistic case (consumers initially overestimate true product quality) is in sharp contrast to our model: in his model, the firm benefits from keeping prices high, because the reduction in demand implies that word about it's true quality spreads slower; in our framework the firm cannot benefit from higher prices, because disappointed consumers induce less favorable beliefs through reviews immediately.

Differences in production costs drive the results in Milgrom and Roberts (1986). Highquality firms choose a price high enough such that the resulting demand reduction makes imitation unprofitable for low-quality firms (see also Bagwell and Riordan (1991)). Even though our framework does not incorporate differences in production costs, low-quality firms exert pressure on high-quality firms to separate themselves in prices. Additionally, in our model there is a pressure from high-quality firms on low-quality firms: The highquality firm may want to imitate a lower price and hence sacrifice early period profit for the sake of more favorable reviews and hence higher profits in the future; an aspect that 
is absent in Milgrom and Roberts (1986).

Consumer reviews serve to reveal information about product quality, which creates a natural link to the literature on certification and disclosure (see Dranove and Jin (2010) for an extensive review of this literature). Albano and Lizzeri (2001) study the effect of a certification intermediary and point out that also a review site may act as such an intermediary. In a model with endogenous product quality choice, a certification intermediary can create incentives to improve quality. We abstract from product quality choice and focus on the strategic choice of prices (and hence creation of expectations) once quality is given already.

The reputation framework of Jullien and Park (2014) is particularly close to our study, in the sense that firms strategically influence consumer expectations. However, as opposed to our model, expectations do not result from prices, but a cheap-talk message of the firm about the quality. In fact, prices are no choice variables at all in their model. As a good reputation is more valuable to a high-ability firm, it will also reveal itself truthfully, but this does not hold for low quality firms. Another difference to our model is that consumer expectations do not influence review creation.

An important concern about online reviews are fraudulent and manipulative reviews, which has been empirically studied by Mayzlin et al. (2014), who find some evidence for potentially fraudulent online reviews. However, they do not model review creation of consumers explicitly. While we acknowledge that review manipulation might effect online reviews, we abstract from manipulation and focus on the reviews created by consumers instead. Chen and Xie (2008) model the informativeness of consumer reviews as a parameter, and find that they can operate as 'sales assistants' to the firm, which in turn might affect the marketing strategy. This interpretation is also consistent with our model; however, in our model informativeness of consumer reviews emerges endogenously through the strategic choice of the firm and not as a parameter.

Our study describes situations in which a firm already obtained an experience good as a result of research and development efforts, and is now faced with the decision of introducing it optimally. The implied trade-off is very different to that of sellers on platforms like eBay, who may engage in high effort initially in order to build up a good a reputation and reap that reputation later by shirking (Jullien and Park, 2014, Klein et al., 2016). We strictly separate the life-cycle of the product into two periods: an introductory 
period in which reviews do not yet carry sufficient information to override the quality signalled through early-period prices; and a second period in which the firm can profit from having gained a high reputation, but importantly cannot 'shirk' or engage in any other action harmful for consumers. The firm simply charges a price which equals the expected quality given the review. As we do not need to be concerned about end-round effects, this framework lends itself naturally to a two-period framework.

Our study also offers a novel explanation why firms might sell at prices below marginal costs in the introductory phase of a product. For instance, in Farrell and Klemperer (2007) low introductory prices are charged in order to lock-in consumers in the future. Low prices may be charged for a certain time period in order to drive out competing firms (predatory pricing). Loss-leaders (Lal and Matutes, 1994) attract consumers to the store in order to also sell other products with higher margins. In our paper, these motives are absent since consumers purchase only once and firms do not enter or exit the market endogeneously. Instead, a low introductory prices charged by low quality types ensure that high quality types do not try to underpromise and overdeliver.

In the next section we lay out the theoretical baseline model with a monopolist seller. In Section 3 we describe the unique monotone D1-satisfying equilibrium of our model. We study comparative statics in Section 4. Section 5 studies an extension to duopoly, and Section 6 concludes.

\section{Model}

There are two periods 1 and 2 where a monopolist sells an experience good. The good is of uncertain quality which is denoted by $q$. Quality is drawn from a twice continuously differentiable distribution $F(q)$ (with associated density $f(q)$ ) over the interval $[\underline{q}, \bar{q}]$ where $\bar{q}>\underline{q} \geq 0$. The constant marginal cost is equal for all qualities. This assumption can be interpreted as a firm which already invested in research and development, and for reasons outside of the scope of the model is marketing a product of quality $q$. Without loss, we normalize the cost to zero. The firm is informed about its quality while consumers are not.

All consumers are identical and value one unit of quality $q$ at $q$, thus assuming that $\underline{q} \geq 0$ implies that even the lowest quality is valued at or above its marginal cost. We 
assume that there is a mass 1 of consumers in the first period and mass $n>0$ in the second period. Consumers are assumed to be risk neutral and buy one unit of the good if its expected quality is equal or above the price. ${ }^{4}$ Consumers observe quality after purchase.

The timing is as follows: $q$ is drawn from $F(q)$ and becomes known to the firm. The firm charges its first period price $p_{1}$ that is observed by first period consumers (but crucially not by second period consumers) who decide to purchase or not. To do so, consumers in the first period form a point belief $q^{e}\left(p_{1}\right) .{ }^{5}$ Consumers then compare $q^{e}\left(p_{1}\right)$ and $p_{1}$ and purchase if their utility is positive.

Second period consumers do not observe price or quality from the first period directly. They do observe a signal $s \in\{g, b\}$ (a good or a bad review) generated by first period consumers, which is also observed by the firm. Having observed the signal, the firm chooses its second period price $p_{2}$. Once consumers observe $p_{2}$ they decide whether to buy or not. After this the game ends.

The probability of a good review is given by the review function $r\left(q, q^{e}\right)$, which depends on first period quality $q$ but also on the belief $q^{e}$. For the moment, we take this reducedform review function and its properties as given, and we provide possible rationales behind it later. Note that the price $p_{1}$ does not influence $r$ directly, but may do so through its effect on $q^{e}$. This assumption is consistent with our premise that reviews depend on consumers' prior expectations, and prices are solely a device through which firms affect expectations. If the good is not sold in the first period, for simplicity, we assume that a bad review is generated for sure.

Throughout this paper, $r_{x}$ denotes the partial derivative of $r$ with respect to $x=q, q^{e}$. For all $q$ and $q^{e}, r\left(q, q^{e}\right) \in[0,1]$ and is a well-behaved twice continuously differentiable function in both arguments with the following properties:

Assumption 1. (i) $r\left(q, q^{e}\right)$ is strictly increasing in $q$; (ii) $r\left(q, q^{e}\right)$ is strictly decreasing in $q^{e}$; (iii) $r(q, q)$ is strictly increasing in $q$; (iv) $r_{q^{e}}(q, q)$ is increasing in $q$; (v) $r_{q^{e} q^{e}} \leq 0$.

(i) implies that holding expected quality fixed, higher quality is more likely to receive a good review. (ii) implies that holding quality fixed, higher expectation of quality reduces

\footnotetext{
${ }^{4}$ In principle, consumer may also randomize when they are indifferent between buying or not, which may introduce additional multiplicity of equilibria.

${ }^{5}$ Dispersed beliefs can also be considered, but are not particularly important for the separating equilibria we derive here.
} 
the probability of a good review. (iii) implies that the probability of a good review is increasing in a correctly anticipated quality $q$, i.e. if consumers expect quality $q$ and get quality $q$, the probability they write a good review is higher the higher is $q$. These three are natural given our premise that reviews convey information, and react positively to the gap between true and expected quality.

The last two assumptions are sufficient to guarantee that the firm's maximization problem is well behaved. In particular, (iv) implies that reduction in the probability of a good review resulting from exaggerating quality is lower the higher the true quality. That is, when a firm induces a higher expectation of quality, the resulting reduction in probability of a good review is is higher in absolute value the lower is the true quality that the firm has. (v) means that reduction in good reviews due to exaggerate quality accelerates as the exaggeration grows. (iv) and (v) are not necessary but are sufficient to guarantee that firm's maximization problem is well-behaved. ${ }^{6}$

Our solution concept is (weak) Perfect Bayesian equilibrium $(\mathrm{PBE})^{7}$ and we use the D1 criterion (Cho and Sobel, 1990) to refine out-of-equilibrium beliefs.

\section{Equilibrium}

Throughout this paper, we focus on separating equilibria since we are interested in the potential of reviews to create incentives to signal quality truthfully.

We shall solve the game with backwards induction. In period 2, conditional on realized signal $s$ there can be no separating equilibrium. This is because all types will have an incentive to charge the highest price charged by any type and sell the good for sure. Thus, there can only be a pooling equilibrium for each $s$ where $p_{2}(s)$ should not exceed $E(q \mid s)$. There will be multiple such equilibria but we will concentrate on the most profitable equilibrium for the firm where

$$
p_{2}^{*}(s)=E(q \mid s) .
$$

\footnotetext{
${ }^{6}$ The necessary condition for the concavity of the firm's profit function in equilibrium is $r_{q^{e}} q^{e}\left(q^{e}, q^{e}\right)+$ $r_{q q^{e}}\left(q^{e}, q^{e}\right)-r_{q^{e} q^{e}}\left(q, q^{e}\right)>0$, where (iv) imposes $r_{q^{e} q^{e}}\left(q^{e}, q^{e}\right)+r_{q q^{e}}\left(q^{e}, q^{e}\right)>0$ and (v) implies $-r_{q^{e} q^{e}}\left(q, q^{e}\right)>0$. We will use (iv) separately to obtain a simple expression for the lower bound on $n$, but indeed could instead of separately assuming (v) simply require a less demanding $\left(r_{q^{e} q^{e}}\left(q^{e}, q^{e}\right)+r_{q q^{e}}\left(q^{e}, q^{e}\right)\right)-r_{q^{e} q^{e}}\left(q, q^{e}\right)>0$ in addition to (iv).

${ }^{7}$ See Mas-Colell et al. (1995).
} 
We shall assume that for all $p_{2} \neq p_{2}^{*}(s)$, consumers form a belief $q^{e}\left(p_{2}\right)=p_{2}-\varepsilon, \varepsilon>0$, and thus do not buy. It is easy to verify that such beliefs satisfy the D1 criterion.

In order to simplify notation, we define the marginal benefit of obtaining a good instead of a bad review as

$$
\Delta \equiv p_{2}^{*}(g)-p_{2}^{*}(b)
$$

Note that if $s$ is uninformative about $q$, then $\Delta=0$. In the proof to Proposition 1 we show that $\frac{\partial r(x, x)}{\partial x}>0$ implies $\Delta>0$.

Now we proceed to the first period. We shall aim to construct a separating equilibrium of the following form: each type $q$ sets a price $p_{1}=p^{*}(q)$ where $p^{*}(\cdot)$ is a twice continuously differentiable strictly increasing function. Given this, all prices lie between $p^{*}(q)$ and $p^{*}(\bar{q})$.

In order to characterize separating equilibria of this form, we have to make sure that no type wants to deviate from the equilibrium. For type $q$, the profit from charging a price $p$ when consumers hold belief $q^{e}(p)$ is

$$
\pi(q, p)=\mathbb{1}_{\left\{q^{e}(p) \geq p\right\}} \cdot\left[p+n \Delta r\left(q, q^{e}(p)\right)\right]+n p_{2}^{*}(b)
$$

where $\mathbb{1}$ is an indicator function. We have a separating equilibrium if $\pi(q, p)$ is maximized at $p(q)$ for all $q$. The beliefs should be such that for all $p \in\left[p^{*}(\underline{q}), p^{*}(\bar{q})\right]$ we should have $q^{e}(p) \geq p$ or else consumers would not buy for such a price, and so the type which were supposed to charge such $p$ would deviate.

In order to find $p^{*}(q)$, first notice that for any $p$ in the support of equilibrium prices, there is a unique belief $q^{e}$ such that $p^{*}\left(q^{e}\right)=p$. Thus for all $p \in\left[p^{*}(q), p^{*}(\bar{q})\right]$ (out-ofequilibrium prices will be dealt with below) we can write

$$
\pi\left(q, q^{e}\right)=p^{*}\left(q^{e}\right)+n\left(p_{2}^{*}(b)+\Delta r\left(q, q^{e}\right)\right)
$$

and assume that type $q$ maximizes its profit by choosing $q^{e} \in[\underline{q}, \bar{q}]$.

We can now use (1) to maximize $\pi\left(q, q^{e}\right)$ with respect to $q^{e}$ and impose $q^{e}=q$ as a necessary condition for a separating equilibrium. This gives

$$
\frac{\partial p^{*}(q)}{\partial q}=-n \Delta r_{q^{e}}(q, q),
$$

from which we obtain

$$
p^{*}(q)=-n \Delta \int_{\underline{q}}^{q} r_{q^{e}}(x, x) d x+C,
$$


where $C$ is a constant.

As we show below, by D1 criterion we will have $p^{*}(\bar{q})=\bar{q}$, which when combined with (3) will pin down the candidate equilibrium price schedule.

For prices outside of $\left[p^{*}(\underline{q}), p^{*}(\bar{q})\right]$ beliefs are assumed to be of the following form: for $p<p^{*}(\underline{q})$ consumers believe that quality is $\underline{q}$, for $p>\bar{q}$ they believe that quality is $\bar{q}$. Deviating to $p<p^{*}(\underline{q})$ is clearly dominated by deviation to $p=p^{*}(\underline{q})$ because both induce the same future demand but $p(\underline{q})$ leads to higher contemporaneous profit. Given $p^{*}(\bar{q})=\bar{q}$ for no $p>p^{*}(\bar{q})$ does a firm make sales in the first and thus the second period. We thus conclude that the only relevant prices are in the support.

In order to ensure that $p^{*}(q)<q$ for all $q$, we will require a lower bound on $n$, which we define as:

$$
\underline{n} \equiv \frac{1}{-\Delta r_{q^{e}}(\bar{q}, \bar{q})}
$$

Given all the above, we can finally state:

Proposition 1. If $n \geq \underline{n}$, then in the unique monotone D1-satisfying separating equilibrium prices are given by

$$
p^{*}(q)=\bar{q}+n \Delta \int_{q}^{\bar{q}} r_{q^{e}}(x, x) d x .
$$

Proof. This and other proofs are in Appendix B.

The structure of the equilibrium in Proposition 1 and the bound on the number of future consumers $n$ highlights the fundamental tension of our model. When $n$ is too low, then low quality types do not care enough about future profits, overpromise and underdeliver in the introductory period, undeterred by relatively small profit losses in the second period. High $n$ also increases the incentive to underpromise and overdeliver for high quality types. This is why the equilibrium price is adjusted downwards when $n$ increases, thus the incentive to underpromise is naturally curtailed by low prices and profits associated with underpromising.

As we detail in the next section, equilibrium first-period prices are inversely related to $n$, and indeed for sufficiently high $n$ prices charge by low quality types are negative (below marginal cost).

The price schedule is concave in $q$, which follows from Assumption 1 part (iv). This is why the condition $p^{*}(q) \leq q$ is most binding for qualities close to $\bar{q}$, and therefore why 
the lower bound on $n$ is in terms of $\bar{q}$. Another interesting feature of the price schedule is that $v(q)=q-p^{*}(q)$ is decreasing in $q$, so that the best value is offered by the worst quality types. Conversely, consumers get the highest utility from the lowest prices in equilibrium. ${ }^{8}$ This is more so, the higher is $n$, as noted above.

We also note here that high $n$ is not sufficient for the separating equilibrium existence unless we have $\Delta>0$ and $r_{q^{e}}<0$. This means that for equilibrium we need that inequilibrium good review probability $r(q, q)$ has to increase in $q$ (which implies $\Delta>0$ ) and the review probability has to change with $q^{e}$ out of equilibrium.

\section{Comparative statics}

Our equilibrium characterization depends entirely on the review generation function $r\left(q, q^{e}\right)$, the distribution of qualities $F(q)$ and number of post-introductory period consumers $n$. In this section we consider how these affect equilibrium structure.

The relationship between $p^{*}(q)$ and $n$ is relatively straightforward: the higher $n$, the lower is the equilibrium price schedule. The reason for this lies in the fact that the price charged by the highest quality is fixed at $\bar{q}$. For high qualities the desire to underpromise and overdeliver (by charging low prices) increases with $n$. As $n$ grows, so does their incentive to deviate, so to discourage it, the equilibrium price schedule is pushed down, more so for the lowest qualities.

The most interesting comparative statics in the model are with regards to the review generating function. It affects the equilibrium price schedule through two channels. First, $r\left(q, q^{e}\right)$ determines the post-introductory price premium from a good review, as captured by $\Delta$. Loosely speaking, the more informative are reviews, the higher is $\Delta$ and so the lower is the price schedule. Informativeness depends on $R(q) \equiv r(q, q)$, the in-equilibrium probability of a good review, rather than the full function $r\left(q, q^{e}\right)$. Second, equilibrium prices depend on how review probability adjusts with expectation of quality via $r_{q^{e}}(q, q)$. In general, this effect is not directly related to $R(q)$. The more responsive are reviews to movements in quality expectations, the lower are prices. Any model that generates $r\left(q, q^{e}\right)$ may affect both of these channels differently and thus result in different predictions about prices.

\footnotetext{
${ }^{8}$ This follows from $n \geq \underline{n}$ and part (iv) of Assumption 1.
} 
More formally, we consider a class of linear in-equilibrium review functions.

Definition 1. A linear in-equilibrium review function is given by

$$
R(q ; \alpha)=a+\alpha(b+q)
$$

where $a>0$ and $b$ are such that $0 \leq R(q ; \alpha) \leq 1$ for all $q \in[\underline{q}, \bar{q}]$ and $\alpha \in[0,1]$.

We can readily link such an in-equilibrium review function to Lehmann informativeness (a formal definition thereof is in the appendix). Intuitively, for a higher $\alpha$ good reviews are increasingly more likely the higher is the quality.

Proposition 2. If the review function $r\left(q, q^{e} ; \alpha\right)$ results in a linear in-equilibrium function $R(q ; \alpha)$ then

i) $E(q \mid g)$ increases in $\alpha, E(q \mid b)$ decreases in $\alpha$, and hence $\Delta$ increases in $\alpha$ and moreover

ii) Lehmann informativeness increases in $\alpha$.

Proof. See appendix.

Combining this consideration with the equilibrium pricing function (5), we have the following general result:

Corollary 1. Consider a review function $r\left(q, q^{e} ; \alpha\right)$ that results in a linear in-equilibrium function $R(q ; \alpha)$. Then increasing $\alpha$ increases Lehmann informativeness, but may increase or decrease equilibrium prices

$$
p^{*}(q ; \alpha)=\bar{q}+n \Delta(\alpha) \int_{q}^{\bar{q}} r_{q^{e}}(x, x ; \alpha) d x
$$

depending on the effect of $\alpha$ on $r_{q^{e}}$.

Proof. Increasing informativeness follows from Proposition 2, which also states that $\Delta(\alpha)$ in (7) increases in $\alpha$, which in turn decreases (7) since the term after it is negative. Changing $\alpha$ also affects $r_{q^{e}}(x, x ; \alpha)$, possibly affecting $p^{*}$ in the other direction as $\Delta$, and hence the net effect on $p^{*}$ is ex-ante unclear.

To illustrate this result, we will consider two possible $r\left(q, q^{e}\right)$ functions. Both will share the same $R(q)$, but will differ dramatically in their comparative statics. To simplify things, for what follows let $F \sim U(1,2)$. 
Example 1. Consider a review generation function of the form

$$
r\left(q, q^{e}\right)=\frac{1}{2}\left(1-\alpha \frac{q^{e}}{q}\right)+\alpha(q-1),
$$

where $\alpha \in[0,1]$. This function satisfies Assumption 1, and has the corresponding linear in-equilibrium review function

$$
R(q)=\frac{1-\alpha}{2}+\alpha(q-1)
$$

The above $R(q)$ has the property that it goes from $\frac{1-\alpha}{2}$ to $\frac{1+\alpha}{2}$ as $q$ goes from 1 to 2. Clearly, as $\alpha$ increases, the signal structure becomes more informative, with $\alpha=0$ providing no information.

In this case an increase in $\alpha$ has two beneficial effects for consumers of the introductory period. First, increasing $\alpha$ increases informativeness, hence $\Delta$ and leads to lower prices. We can solve for $\Delta=\frac{\alpha}{3}$, so indeed increasing $\alpha$ increases post-introductory period price gain from a good review. Second, increasing alpha increases how much reviews react to $q^{e}$ and thus also reduces prices through this channel. We can find

$$
\int_{q}^{\bar{q}} r_{q^{e}}(x, x) d x=-\frac{\alpha}{2} \log (2 / q),
$$

with means that increasing $\alpha$ also increases how responsive reviews are to expected quality.

We thus conclude that both channels through which $\alpha$ affects the price schedule work in the same direction, and so increasing $\alpha$ reduces prices. Indeed, from Proposition 1 we can find

$$
p^{*}(q)=2-\frac{\alpha^{2} n}{6} \log (2 / q)
$$

and so one can verify directly that prices are inversely related to $\alpha$, as well as $n .{ }^{9}$ As explained above, for $n$ sufficiently high, prices of low quality types, e.g., $q=\underline{q}$, are below zero. Namely, for $n>\frac{12}{\alpha^{2} \log (2)}$ there exist types close to 1 which in equilibrium charge negative prices.

The equilibrium price schedules are illustrated in Figure 1 for $n=15$ and $n=25$, where the dashed line depicts $p(q)=q$. One can see that for $n=25$ quality types close to $\underline{q}=1$ charge negative prices in the introductory period.

\footnotetext{
${ }^{9}$ For the equilibrium to exist, we need $n \geq \underline{n}=\frac{12}{\alpha^{2}}$.
} 


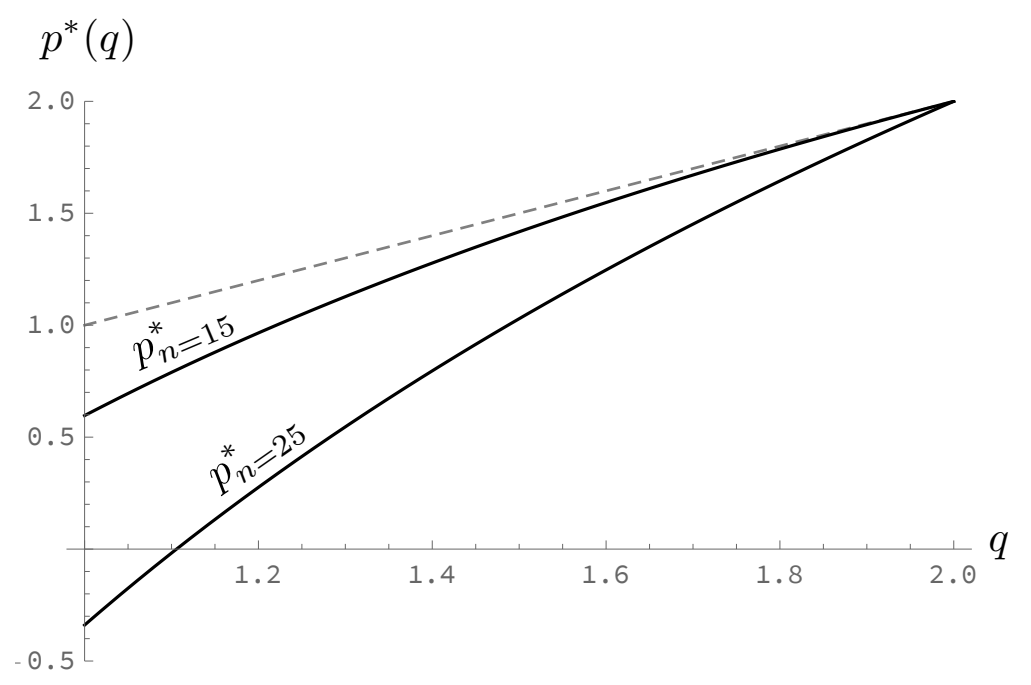

Figure 1: Equilibrium price schedules for $\alpha=0.9, n=15$ and $n=25$. Price schedule may never go above the dashed line where $p(q)=q$.

Example 2. We now turn to our second example where $\alpha$ governs how much weight is put on true quality rather than expected quality. Let the review function be

$$
r\left(q, q^{e}\right)=\frac{1}{2}(1-3 \alpha)+\alpha q+(1-\alpha)\left(1-\frac{q^{e}}{q}\right)
$$

One can interpret this $r\left(q, q^{e}\right)$ as originating from consumers who report quality (their influence increases with $\alpha$ ) and those who report value obtained from the good (the difference between quality and price payed), whose influence diminishes with $\alpha$. We think of quality reporters as consumers whose review is primarily based on observable product characteristics, whereas value reporters also take into account additional, possibly changing, attributes, like for example prices or prior expectations.

This review function generates the same equilibrium signals as our first example because it share the same $R(q)$. This means that $\Delta=\frac{\alpha}{3}$ as before, and thus increasing $\alpha$ increases price premium that a good signal commands. However, now increasing $\alpha$ reduces how much reviews react to quality expectations, namely when $\alpha=1$ signal is entirely independent of $q^{e}$. In terms of our price schedule, $\int_{q}^{\bar{q}} r_{q^{e}}(x, x) d x=-(1-\alpha) \log (2 / q)$, so increasing $\alpha$ reduces the effect of $q^{e}$ on equilibrium.

Since this review generation function satisfies Assumption 1, we can use Proposition 1 to compute

$$
p^{*}(q)=2-\frac{1}{3}(1-\alpha) \alpha n \log (2 / q)
$$

where we require $n \geq \underline{n}=\frac{6}{(1-\alpha) \alpha}$ for equilibrium to exist. 
Unlike our first example, here equilibrium prices are non-monotone in $\alpha$, decreasing in $\alpha$ for $\alpha<\frac{1}{2}$ and increasing for $\alpha>\frac{1}{2}$. This is because for low $\alpha$ the informativeness effect through $\Delta$ dominates, whereas for high $\alpha$ the loss of responsiveness to expectations of quality starts to push prices up. We thus conclude that increasing the influence of quality reporters initially reduces prices, but as their influence grows, eventually prices increase the more accurately quality is reported.

Figure 2 shows equilibrium prices and profits as a function of $\alpha$. We set $n=\frac{6}{0.7 \times 0.3}$ and constrain $\alpha \in[0.3,0.7]$ so that $n \geq \underline{n}$. Price schedules in the right panel show the non-monotonicity explained above. While price is flat for $q=\bar{q}=2$, it is non-monotone for $q=1$. The same is true for exa ante expected price $E p^{*}=\int_{\underline{q}}^{\bar{q}} p^{*}(q) f(q) d q$. In the left panel we depict total (including both periods) equilibrium profits. Profits are increasing in $\alpha$ for $q=2$ and decreasing for $q=1$. This is because for $q=2$ more informative reviews increase $\Delta$ and $q=2$ is more likely to get a good review, the reverse is true for $q=1$. Ex ante profits given by $E \pi^{*}=\int_{\underline{q}}^{\bar{q}} \pi^{*}(q) f(q) d q$ are non-monotone. This is because post-introductory period profits are ex ante independent of $\alpha$ (the firm charges ex post expected quality regardless of informativeness). Therefore, total profits ex ante will follow ex ante price, which is non-monotone. ${ }^{10}$
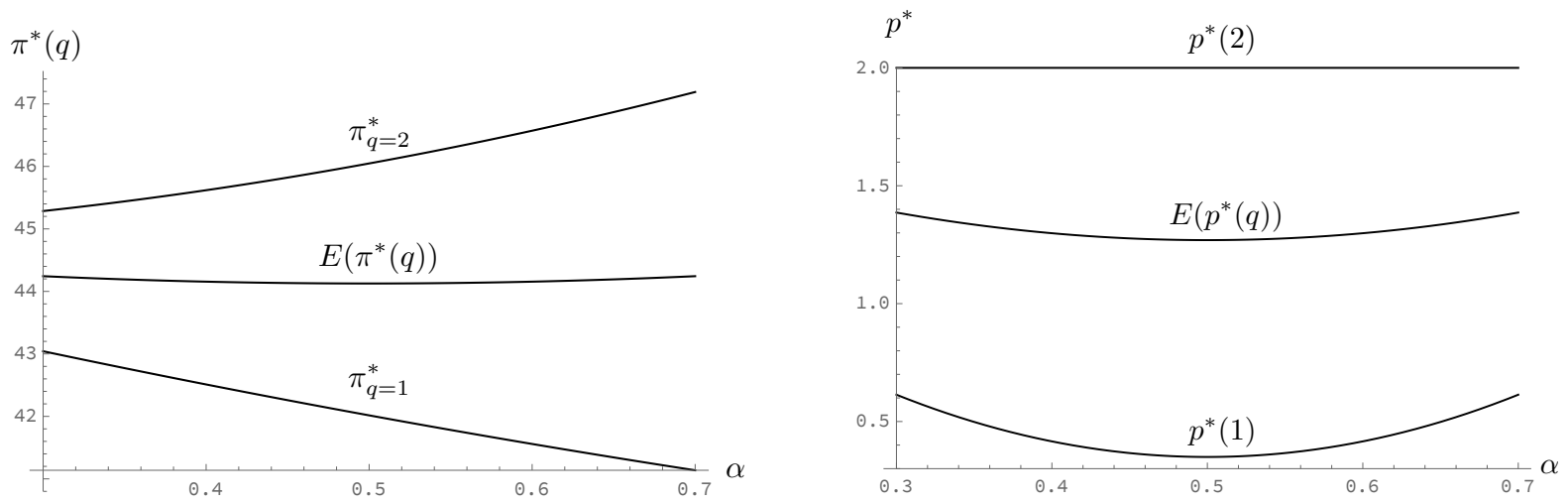

Figure 2: Comparative statics with respect to $\alpha$ for profits (left) and prices (right).

\footnotetext{
${ }^{10} E p^{*}=2-\frac{1}{3}(1-\alpha) \alpha n(1-\log (2))$, which just like $p^{*}(q)$ is increasing (decreasing) in $\alpha$ for $\alpha>1 / 2$ $(\alpha<1 / 2)$.
} 


\section{Duopoly}

We now show that the key intuition developed in the previous sections with a monopolist firm also carries over with competition. We will focus on the duopoly case with $m=2$ firms here, but intuitions translate to oligopolistic setting with $m>2$.

Let quality for each of the two firms be drawn independently from $F(q)$ over $[q, \bar{q}]$, and assume that $q_{i}$ is firm $i$ 's private information. We further assume that $F(q)$ satisfies $\bar{q}-q \leq \frac{1-F(q)}{f(q)}$ for $\forall q \in[\underline{q}, \bar{q}] .{ }^{11}$ This assumption will become important for the existence of the separating equilibrium.

We maintain the previous assumptions that there are two periods and introductoryperiod consumers who purchase from firm $i$ create a noisy signal $s_{i} \in\{g, b\}$ according to a probabilistic review function $r\left(q, q^{e}\right)$, which satisfies Assumption 1. As before, we will assume that if consumers don't buy in the first period, then the signal is $b$.

In contrast to the monopoly model, in the first period in addition to measure 1 of consumers, there is now also a (possibly negligible) positive measure $\lambda$ of loyal consumers per firm, who consider only that firm, and buy if $q^{e} \geq p .{ }^{12}$ To facilitate comparisons with the monopoly model, we will assume that the number of second period consumers is $(1+\lambda) n$ so that for each firm the ratio of demand in the second period to the total possible demand in the first period is $n$ as before.

Following a related model by Janssen and Roy (2010), and in line with our monopoly model, we will construct a symmetric separating equilibrium in which in the first period, only the firm with the lowest price (and quality level) of the two sells to non-loyal consumers. In such equilibrium $v(q)=q-p^{*}(q)$ will be decreasing in $q$, as it is in the monopoly model. We will first construct such an equilibrium and then will impose conditions on primitives that guarantee $v^{\prime}(q)<0$.

As before, we start from the post-introductory period. It is clear that if both firms draw the same signal, consumers have no information to differentiate the two and so price competition will result in zero profits a la Bertrand. However, upon consumers receiving one good and one bad signal, they will compute expected quality difference based on the signal, denoted again by $\Delta$ and computed as before. Firms will have no scope for signaling

\footnotetext{
${ }^{11}$ This condition holds when a density function is non-decreasing, e.g., for the uniform distribution.

${ }^{12}$ In the absence of loyal consumers, the highest quality levels would never sell at all in the first period, resulting in no review for one of the firms and potential equilibrium indeterminacy.
} 
in the second period, and so in the equilibrium upon receiving differing signals, the firm with a good signal will charge a price equal to $\Delta$ and serve all the consumers, while the firm with a bad signal will charge 0 . For this to be true we assume that when indifferent consumers purchase from the firm selling higher quality.

Given that consumer are homogeneous, and that $v(q)$ is decreasing in $q$, if a firm induces first period consumers to believe its quality is $q^{e}$, then it only sells to non-loyal consumers if the other firm has $q>q^{e}$, which occurs with probability $1-F\left(q^{e}\right)$.

The probability that the other firm gets a bad review is given by

$$
\beta \equiv \int_{\underline{q}}^{\bar{q}}(1-R(x)) f(x) d x .
$$

Thus, in a separating equilibrium, for prices in the equilibrium support, profits of a firm can be written as:

$$
\pi(p)=p\left(\lambda+1-F\left(q^{e}(p)\right)\right)+n(1+\lambda) \Delta \beta r\left(q, q^{e}(p)\right)
$$

Since in equilibrium each price uniquely pins down $q^{e}=p^{-1}(p)$, for $q^{e} \in[\underline{q}, \bar{q}]$ this can equivalently be written as

$$
\pi\left(q^{e}\right)=p\left(q^{e}\right)\left(\lambda+1-F\left(q^{e}\right)\right)+n(1+\lambda) \beta \Delta r\left(q, q^{e}\right)
$$

For now, we will assume that the profit function is concave in equilibrium for all $q^{e}$, and will later show that Assumption 1 suffices for this.

Taking FOC with respect to $q^{e}$ yields the following linear first order differential equation

$$
p^{\prime}\left(q^{e}\right)\left(\lambda+1-F\left(q^{e}\right)\right)-p\left(q^{e}\right) f\left(q^{e}\right)+n(1+\lambda) \Delta \beta r_{q^{e}}\left(q, q^{e}\right)=0,
$$

with a unique (up to a constant $C$ ) solution:

$$
p^{d}(q)=\frac{C-n(1+\lambda) \Delta \beta \int_{\underline{q}}^{q} r_{q^{e}}(x, x) d x}{1+\lambda-F(q)} .
$$

In line with the monopoly model, the unique monotone D1 separating equilibrium will have $p^{d}(\bar{q})=\bar{q}$, which when combined with the equation above pins down the equilibrium candidate schedule.

As before, we may need to impose a lower bound on $n$. The lower bound may be superfluous here, unlike the monopoly model where it always binds, because prices tend 
to be lower under duopoly. We define the lower bound as

$$
\underline{n}^{d} \equiv \max _{q \in[q, \bar{q}]} \frac{f(q)\left(\lambda \bar{q}+(\bar{q}-q) r_{q^{e}}(q, q)\right)-(1+\lambda-F(q))^{2}}{\beta \Delta r_{q^{e}}(q, q)(1+\lambda-F(q))(1+\lambda)},
$$

which, as explained before, may be negative, and thus non-binding.

We can now state

Proposition 3. If $n \geq \underline{n}^{d}$, then a D1-satisfying, monotone, symmetric separating equilibrium of the duopoly model exists. In this equilibrium prices are given by

$$
p^{d}(q)=\frac{\lambda \bar{q}+(1+\lambda) n \Delta \beta \int_{q}^{\bar{q}} r_{q^{e}}(x, x) d x}{1+\lambda-F(q)} .
$$

Proof. See appendix.

It is clear from the above proposition why we require positive measure of loyals $\lambda$. In their absence the highest quality type would earn zero profits in the first period and thus in order to separate all other types would have to charge negative prices.

We can see that the duopoly model is most comparable to the monopoly model when $\lambda=1$, so that the highest type makes the same first-period profit in both models. The duopoly price schedule with $\lambda=1$ is given by

$$
p^{d}(q)=\frac{1}{2-F(q)}\left(\bar{q}+2 n \Delta \beta \int_{q}^{\bar{q}} r_{q^{e}}(x, x) d x\right) .
$$

Recall that the monopoly price schedule takes a very similar form

$$
p^{*}(q)=\bar{q}+n \Delta \int_{q}^{\bar{q}} r_{q^{e}}(x, x) d x .
$$

We can see that under duopoly the introductory-period price for $q<\bar{q}$ can be higher or lower depending on parameters. On the one hand, positive (negative) duopoly prices are pushed down (up) by the term $\frac{1}{2-F(q)}>0$, but on the other hand, they can be pushed up if $\beta<\frac{1}{2}$. For $\beta \geq \frac{1}{2}$, for all $q$ that charge positive prices, we have $p^{*}(q)>p^{d}(q)$. A particularly simple situation occurs when the probability of a good review, $\beta=\frac{1}{2}$.

To gain more insights into the comparison between monopoly and duopoly, we revisit our Example 1 where indeed $\beta=\frac{1}{2}$.

Example 3. Let everything be the same as in Example 1, namely $q$ uniformly distributed on $[1,2]$ and $R(q)$ given in (9). For this $R(q), \beta=\frac{1}{2}$ so the comparison between the two models is particularly simple for $\lambda=1$ because

$$
p^{d}(q)=\frac{1}{2-F(q)} p^{*}(q) .
$$


From the above equation we can simply conclude that $p^{d}(q)<p^{*}(q)$ for all $q$ such that $p^{d}(q)>0$ and $p^{d}(q)>p^{*}(q)$ for all $q$ (if any) such that $p^{d}(q)<0$. That is, under duopoly, prices are lower when they exceed marginal cost, but are higher when they are below marginal cost. Intuitively, when $\beta=\frac{1}{2}$ considerations regarding future profits are the same for a monopolist and duopolists, except that duopolists are less likely to win competition in the first period, thus for them second-period profits weigh more and push prices down. This logic is reversed for negative prices because, when a duopolist deviates downwards from already negative price, as compared to the monopolist, duopolist suffers additional losses due to increased probability of 'winning' demand (and suffering losses), so price does not have to decrease as much to keep a duopolist from deviating.

Figure 3 replicates Figure 1 for $n=25$ for the monopoly case but adds the duopoly price schedule. Both models are derived for the review function in (8) and $q$ uniformly distributed on $[1,2]$. As can be seen, the equilibrium price is lower for monopoly model for low qualities but higher for high qualities, and the threshold quality delimiting the two cases is where price is 0 .

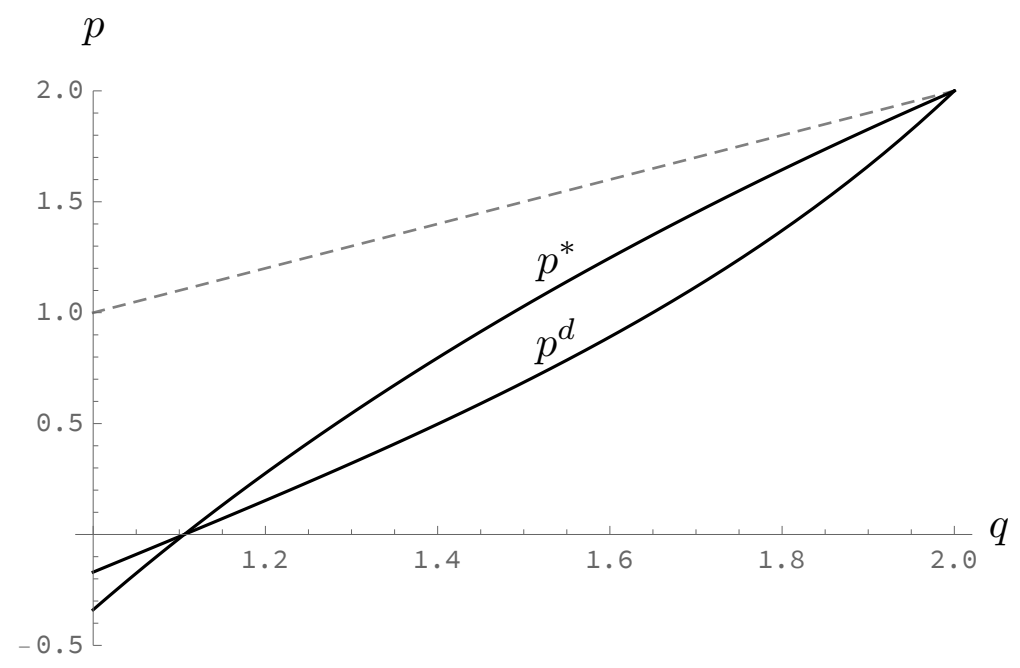

Figure 3: Equilibrium price schedules for monopoly and duopoly.

The exact conditions for equilibrium existence and the equilibrium pricing rule (12) differs from the monopoly setting, however, the key intuition behind separating equilibrium construction, as well as the economic forces behind it, remain the same. The threat of bad reviews disciplines firms of low quality to signal their quality truthfully. At the same time, high quality firms may potentially underpromise and overdeliver in order to get better reviews. In equilibrium, prices are chosen such that deviations from high as 
well as low quality types is deterred. This is only true when $n$ is sufficiently high as to discipline low types, but at the same time prices charged by lower types decrease with $n$ so the higher types do not attempt to underpromise and overdeliver either.

\section{Conclusion}

Our model describes a novel mechanism explaining why consumer review creation is valuable for firms, and how firms can strategically introduce an experience good in order to induce favorable reviews. A firm facing the decision of an introductory price needs to take into account that precisely because a higher price causes higher expectations, consumers are willing to pay more for the product, but importantly are also less likely to write a good review. Anticipating future demand for the product, the firm's choice needs to balance between profits in the introductory and in the mature phase.

Our model specification with quality reporters and value reporters reveals an important tension between the interests of early and future consumers. Indeed, review generation of early consumers might actually harm them because of the incentives this creates for firms. Consumers might be better off if they were able to commit to generate a review in a certain way. Moreover, the extend to which information is transmitted in the beginning might also be limited by subsequent review creation. When punishment for upward deviations becomes too small, or the reward for underpromising and overdelivering too strong, then a separating equilibrium with perfect information transmission in the introductory period may no longer exist.

In the separating equilibrium of our model, higher quality firms charge higher prices. This is also predicted by standard models of vertical differentiation with perfect information. Our model differs from these models since it also provides a rationale for charging different prices during the introductory and the mature phase of a product. Additionally, our model predicts that prices should be lower in markets where more consumers obtain information through reviews, a channel that is absent in standard models. Empirical analysis of these results is left for future research. 


\section{References}

Albano, G. L. and Lizzeri, A. (2001). Strategic certification and provision of quality. International Economic Review, 42(1):267-283.

Anderson, M. and Magruder, J. (2012). Learning from the crowd: Regression discontinuity estimates of the effects of an online review database. The Economic Journal, 122(563):957-989.

Athey, S. and Levin, J. (2001). The value of information in monotone decision problems.

Bagwell, K. and Riordan, M. H. (1991). High and declining prices signal product quality. The American Economic Review, pages 224-239.

Chen, Y. and Xie, J. (2008). Online consumer review: Word-of-mouth as a new element of marketing communication mix. Management Science, 54(3):477-491.

Chevalier, J. A. and Mayzlin, D. (2006). The effect of word of mouth on sales: Online book reviews. Journal of marketing research, 43(3):345-354.

Cho, I.-K. and Sobel, J. (1990). Strategic stability and uniqueness in signaling games. Journal of Economic Theory, 50(2):381-413.

Dranove, D. and Jin, G. Z. (2010). Quality disclosure and certification: Theory and practice. Journal of Economic Literature, 48(4):935-63.

Farrell, J. and Klemperer, P. (2007). Coordination and lock-in: Competition with switching costs and network effects. Handbook of industrial organization, 3:1967-2072.

Janssen, M. C. and Roy, S. (2010). Signaling quality through prices in an oligopoly. Games and Economic Behavior, 68(1):192-207.

Jin, G., Lee, J., Luca, M., et al. (2018). Aggregation of consumer ratings: an application to yelp. com. Quantitative Marketing and Economics, 16(3):289-339.

Jullien, B. and Park, I.-U. (2014). New, like new, or very good? reputation and credibility. The Review of Economic Studies, 81(4):1543-1574.

Klein, T. J., Lambertz, C., and Stahl, K. O. (2016). Market transparency, adverse selection, and moral hazard. Journal of Political Economy, 124(6):1677-1713. 
Lal, R. and Matutes, C. (1994). Retail pricing and advertising strategies. Journal of business, pages 345-370.

Lehmann, E. (1988). Comparing location experiments. The Annals of Statistics, pages $521-533$.

Luca, M. (2016). Reviews, reputation, and revenue: The case of yelp. com. Com (March 15, 2016). Harvard Business School NOM Unit Working Paper, (12-016).

Mas-Colell, A., Whinston, M. D., Green, J. R., et al. (1995). Microeconomic theory, volume 1. Oxford university press New York.

Mayzlin, D., Dover, Y., and Chevalier, J. (2014). Promotional reviews: An empirical investigation of online review manipulation. American Economic Review, 104(8):242155.

Milgrom, P. and Roberts, J. (1986). Price and advertising signals of product quality. The Journal of Political Economy, pages 796-821.

Quah, J. K.-H. and Strulovici, B. (2009). Comparative statics, informativeness, and the interval dominance order. Econometrica, 77(6):1949-1992.

Shapiro, C. (1983). Optimal pricing of experience goods. The Bell Journal of Economics, pages $497-507$. 


\section{Appendix}

\section{A Lehmann's informativeness criterion}

For comparative statics, we need to be able to compare different information structures resulting from different review generation processes, and hence equilibrium information transmission. When we consider parameter variations such that a separating equilibrium exists there is always perfect information provision in the first period. In our reduced-form representation of long-term profits in period two, consumers always pay the expected price (conditional on the signal), so the signal structure is welfare-neutral for second-period consumers.

In order to still differentiate between information provision to second-period consumers, we rank information structures resulting from a separating equilibrium and review generation according to Lehmann's informativeness criterion (Lehmann, 1988, Quah and Strulovici, 2009, Athey and Levin, 2001). This concept is particularly appealing in our environment and is very tractable with our binary signal structure.

Informativeness is defined exclusively in terms of the (linear) in-equilibrium review function $R(q ; \alpha)$. In order to apply Lehmann's informativess criterion, we use an informationpreserving transformation of our binary signal to a continuous random variable which is distributed according to a c.d.f. $\left.G_{(} z \mid q ; \alpha\right)$ for $z \in[0,1]$. The transformation (Lehmann, 1988, Athey and Levin, 2001) works by defining the resulting conditional distribution under $\alpha$ as $G(z \mid q ; \alpha)$ for $z \in[0,1]$ :

$$
G(z \mid q ; \alpha)= \begin{cases}2 z(1-R(q ; \alpha)) & z \leq 1 / 2 \\ 1-2 R(q ; \alpha)(1-z) & z>1 / 2\end{cases}
$$

Under this transformation, a signal $z \leq 1 / 2$ corresponds to a bad review, and a signal $z>1 / 2$ to a good review. Crucially, the signals are uniformly distributed on $[0,1 / 2]$ and $(1 / 2,1]$, respectively. Therefore the exact realization carries no additional information other than whether it was below or above $1 / 2$. This is indeed information preserving. The densities implied by (13) are given by

$$
g(z \mid q ; \alpha)= \begin{cases}2(1-R(q ; \alpha)) & z \leq 1 / 2 \\ 2 R(q ; \alpha)) & z>1 / 2\end{cases}
$$


and hence

$$
\begin{aligned}
E(q \mid z, z \leq 1 / 2) & =\frac{\int_{\underline{q}}^{\bar{q}} q g(z \mid q ; \alpha) d F(q)}{\int_{\underline{q}}^{\bar{q}} g(z \mid q ; \alpha) d F(q)} \\
& =\frac{2 \int_{\underline{q}}^{\bar{q}} q(1-R(q ; \alpha)) d F(q)}{2 \int_{\underline{q}}^{\bar{q}}(1-R(q ; \alpha)) d F(q)} \\
& =E(q \mid b)
\end{aligned}
$$

and analogously it holds that $E(q \mid z, z>1 / 2)=E(q \mid g)$.

We can now give a formal definition of informativeness.

Definition 2. Given two signal structures $\alpha$ and $\alpha^{\prime}$, define $G(T(z, q) \mid q ; \alpha)=G\left(z \mid q ; \alpha^{\prime}\right)$. $\alpha$ is more informative than $\alpha^{\prime}$ if $T(z, q)$ is increasing in $q$ for all $z \in[0,1]$.

The intuition behind this criterion is as follows (Quah and Strulovici, 2009). Fix a signal $z$ from $G\left(z \mid q ; \alpha^{\prime}\right)$. Suppose that as we increase $q$ that generates $z$ from $G\left(z \mid q ; \alpha^{\prime}\right)$, also the corresponding signal from $G(z \mid q ; \alpha)$ increases. In other words, it becomes increasingly more likely that a high signal is generated from a high quality $q$ under $\alpha$. Thus $\alpha$ is more informative.

\section{B Proofs}

The following lemma will be used to prove Proposition 1.

Lemma 1. i) Regardless of beliefs, no type wants to deviate to a price $p$ with $p<p^{*}(\underline{q})$.

ii) By D1 criterion, $p^{*}(\bar{q})=\bar{q}$, thus no type wants to deviate to $p>p^{*}(\bar{q})$.

Proof. i) In terms of post-introductory period profits the most favorable belief is that $q=\underline{q}$. Even if consumers hold such belief when observing $p<p^{*}(\underline{q})$, no firm will fish to deviate to $p$ because each one would instead deviate to $p^{*}(\underline{q})$ which induces the same belief (and thus post-introductory period profits) and earns higher profit in the first period.

ii) Assume the opposite so that $p^{*}(\bar{q})<\bar{q}$. First notice that type $\bar{q}$ always wants to deviate to any price $p^{\prime}>p^{*}(\bar{q})$ unless consumers do not buy at such a price. This is because $\bar{q}$ in equilibrium is already facing least favorable beliefs and thus cannot worsen its future profits by deviating. Thus for this to be a separating equilibrium, it has to be that for all $p^{\prime} \in\left(p^{*}(\bar{q}), \bar{q}\right]$ consumers hold belief $q^{e}\left(p^{\prime}\right)<p^{\prime}$. By D1, the set of types that consumers associate with $p^{\prime}$ should be such that for each such type the set of best 
responses that induce deviation is equal and larger than for all non-included types. For $\bar{q}$, any $q^{e}\left(p^{\prime}\right) \geq p^{\prime}$ induces a deviation, thus for any $p^{\prime}$ the set of beliefs that should induce deviation for an admissible belief should include $\bar{q}$. Because deviations are less profitable the higher is the belief $q^{e}$, D1 criterion admits a type if it has a profitable deviation to $p^{\prime}$ for a belief $q^{e}=\bar{q}$. Now take $p^{\prime}$ arbitrarily close to $p^{*}(\bar{q})$. Because no type wants to deviate to $p^{*}(\bar{q})$, and profits are concave in $q^{e}$, the set of types that have an incentive to deviate to $p^{\prime}$ consists of types that are close to $\bar{q}$. Thus for $p^{\prime}$ it is the case that any D1 admissible belief induces consumers to purchase (all the types that consumers may assign to a deviation to $p^{\prime}$ are arbitrarily close to $\bar{q}$ whereas $p^{\prime}$ is arbitrarily close $p^{*}(\bar{q})$, so by $p^{*}(\bar{q})<\bar{q}$, all such types are above $\left.p^{*}(\bar{q})\right)$. It follows that under beliefs that satisfy D1, $\bar{q}$ wants to deviate, hence no separating equilibrium exists where $p^{*}(\bar{q})<\bar{q}$. Given $p^{*}(\bar{q})=\bar{q}$, a deviation to $p>p^{*}(\bar{q})$ cannot lead to any sales because even under $q^{e}=\bar{q}$ we have $p>q^{e}$.

Proof of Proposition 1. For (5) to be an equilibrium, we need $p^{*}(q) \leq q$ for all $q$ (otherwise consumers don't buy). Before we derive conditions for this to hold, let us assume they do hold and apply the D1 criterion to out-of-equilibrium beliefs to evaluate deviations to $p \notin\left[p^{*}(\underline{q}), p^{*}(\bar{q})\right]$. By Lemma 1 we have that in a D1-satisfying separating equilibrium, $p^{*}(\bar{q})=\bar{q}$.

Once we establish that we should have $p^{*}(\bar{q})=\bar{q}, C$ can be found from (2) as

$$
C=\bar{q}+n \Delta \int_{\underline{q}}^{\bar{q}} r_{q^{e}}(x, x) d x,
$$

so that the equilibrium pricing function is

$$
p^{*}(q)=\bar{q}+n \Delta \int_{q}^{\bar{q}} r_{q^{e}}(x, x) d x .
$$

We now have to impose $p^{*}(q) \leq q$. Rewriting gives

$$
n \geq \frac{\bar{q}-q}{-\Delta \int_{q}^{\bar{q}} r_{q^{e}}(x, x) d x},
$$

which should hold for all $q$, thus we need

$$
n \geq \max _{q \in[\underline{q}, \bar{q}]} \frac{\bar{q}-q}{-\Delta \int_{q}^{\bar{q}} r_{q^{e}}(x, x) d x} .
$$

Taking derivative of the right-hand side of the inequality with respect to $q$ gives

$$
\frac{\int_{q}^{\bar{q}} r_{q^{e}}(x, x) d x-(\bar{q}-q) r_{q^{e}}(q, q)}{\Delta\left(\int_{q}^{\bar{q}} r_{q^{e}}(x, x) d x\right)^{2}} .
$$


By Assumption 1 part (iv) $r_{q^{e}}(q, q)$ is increasing in $q$, so the above derivative is positive because

$$
\frac{\int_{q}^{\bar{q}} r_{q^{e}}(x, x) d x}{\bar{q}-q}>r_{q^{e}}(q, q)
$$

We therefore conclude that

$$
\frac{\bar{q}-q}{-\Delta \int_{q}^{\bar{q}} r_{q^{e}}(x, x) d x}
$$

is increasing in $q$ and thus reaches its maximum at $q=\bar{q}$. Using L'Hospital's Rule we find

$$
\max _{q \in[\underline{q}, \bar{q}]} \frac{\bar{q}-q}{-\Delta \int_{q}^{\bar{q}} r_{q^{e}}(x, x) d x}=\lim _{q \rightarrow \bar{q}} \frac{\bar{q}-q}{-\Delta \int_{q}^{\bar{q}} r_{q^{e}}(x, x) d x}=\frac{1}{-\Delta r_{q^{e}}(\bar{q}, \bar{q})},
$$

So $\underline{n}=\frac{1}{-\Delta r_{q} e(\bar{q}, \bar{q})}$.

Once we have a separating equilibrium, we can find $\Delta$. For a given $q$, the probability of a good review for $q^{e}=q$ is $r(q, q)$. Let

$$
R(q) \equiv r(q, q)
$$

denote the in-equilibrium probability of a good review for quality $q$.

By Bayes rule, conditional on $s=g$, the density of quality being equal to $q$, denoted by $h(q \mid g)$, is given by

$$
h(q \mid g)=\frac{R(q) f(q)}{\int_{\underline{q}}^{\bar{q}} R(x) f(x) d x} .
$$

Using the same method, the density of $q$ given $s=b$, denoted by $h(q \mid b)$ can be written as

$$
h(q \mid b)=\frac{(1-R(q)) f(q)}{\int_{\underline{q}}^{\bar{q}}(1-R(x)) f(x) d x} .
$$

We can now calculate

$$
E(q \mid g)=\int_{\underline{q}}^{\bar{q}} q h(q \mid g) d q \text { and } E(q \mid b)=\int_{\underline{q}}^{\bar{q}} q h(q \mid b) d q,
$$

which combined give

$$
\Delta=E(q \mid g)-E(q \mid b)=\int_{\underline{q}}^{\bar{q}} q(h(q \mid g)-h(q \mid b)) d q .
$$

Using $R^{\prime}(q)>0$ we can show that $\Delta>0$. For this it suffices to show that $h(q \mid g)$ first-order stochastically dominates $h(q \mid b)$. For this, for any $q<\bar{q}$ we need

$$
\frac{\int_{\underline{q}}^{q} R(x) f(x) d x}{\int_{\underline{q}}^{\bar{q}} R(x) f(x) d x}<\frac{\int_{\underline{q}}^{q}(1-R(x)) f(x) d x}{\int_{\underline{q}}^{\bar{q}}(1-R(x)) f(x) d x}
$$


which can be written as

$$
\frac{\int_{\underline{q}}^{q} R(x) f(x) d x}{\int_{\underline{q}}^{\bar{q}} R(x) f(x) d x}<\frac{F(q)-\int_{\underline{q}}^{q} R(x) f(x) d x}{1-\int_{\underline{q}}^{\bar{q}} R(x) f(x) d x} .
$$

The latter can be rewritten as

$$
\int_{q}^{\bar{q}} \frac{f(x)}{1-F(q)} R(x) d x>\int_{\underline{q}}^{\bar{q}} f(x) R(x) d x .
$$

The right hand side of the above is the (unconditional) mean of $R(x)$, the left hand side is the conditional mean of $R(x)$ for $x>q$, which given that $R^{\prime}(x)$ is an increasing function has to always hold, thus we conclude that $\Delta>0$.

From (1) we can also obtain the necessary second-order conditions (SOC) by taking the second derivative with respect to $q^{e}$. The SOC is

$$
\frac{\partial^{2} \pi\left(q, q^{e}\right)}{\partial q^{e 2}}=p^{\prime \prime}\left(q^{e}\right)+n \Delta r_{q^{e} q^{e}}\left(q, q^{e}\right)
$$

now we use (2) to obtain $p^{\prime \prime}\left(q^{e}\right)=-n \Delta r_{q q^{e}}\left(q^{e}, q^{e}\right)-n \Delta r_{q^{e} q^{e}}\left(q^{e}, q^{e}\right)$, which gives

$$
\frac{\partial^{2} \pi\left(q, q^{e}\right)}{\partial q^{e 2}}=-n \Delta\left(r_{q^{e} q^{e}}\left(q^{e}, q^{e}\right)-r_{q^{e} q^{e}}\left(q, q^{e}\right)+r_{q q^{e}}\left(q^{e}, q^{e}\right)\right)<0
$$

This condition holds by combining parts (iv) and (v) of Assumption 1. Thus, each type prefers charging $p(q)$ to any other price in the equilibrium support. Since charging $p(\bar{q})=\bar{q}$ guarantees positive profits in both the first and the second period, it follows that each type makes positive profits in equilibrium, and hence also prefers to participate in the market to start with.

Proof of Proposition 2. i) Lehmann informativeness to increase in $\alpha$ requires that $T(z, q)$ is increasing in $q$ for all $z \in[0,1]$, where $T(z, q)$ is given as the solution to $G_{\alpha}(T(z, q) \mid q)=$ $G_{\alpha^{\prime}}(z \mid q)$ where $\alpha>\alpha^{\prime}$. Since $G$ is defined piecewise in (13), we need to evaluate four different cases:

$$
T(z, q)= \begin{cases}z \frac{1-R\left(q ; \alpha^{\prime}\right)}{1-R(q ; \alpha)} & z \leq 1 / 2, z \frac{1-R\left(q ; \alpha^{\prime}\right)}{1-R(q ; \alpha)} \leq 1 / 2 \\ \frac{-1+2 R(q ; \alpha)-2\left(-1+R\left(q ; \alpha^{\prime}\right)\right) z}{2 R(q ; \alpha)} & z \leq 1 / 2, z \frac{1-R\left(q ; \alpha^{\prime}\right)}{1-R(q ; ;)}>1 / 2 \\ \frac{1+2 R\left(q ; \alpha^{\prime}\right)(-1+z)}{2-2 R(q ; \alpha)} & z>1 / 2, \frac{1+2 R\left(q ; \alpha^{\prime}\right)(-1+z)}{2-2 R(q ; \alpha)} \leq 1 / 2 \\ \frac{R(q ; \alpha)+R\left(q ; \alpha^{\prime}\right)(-1+z)}{R(q ; \alpha)} & z>1 / 2, \frac{1+2 R\left(q ; \alpha^{\prime}\right)(-1+z)}{2-2 R(q ; \alpha)}>1 / 2\end{cases}
$$


Given $\alpha>\alpha^{\prime}$, we find that all of these expressions increase in $q$ for all $z$, and hence that Lehmann informativeness increases in $\alpha$.

ii) We first show that $E(q \mid g)$ increases in $\alpha . E(q \mid g)$ is defined as

$$
E(q \mid g)=\frac{\int_{\underline{q}}^{\bar{q}} q R(q ; \alpha) d F(q)}{\int_{\underline{q}}^{\bar{q}} R(q ; \alpha) d F(q)}
$$

and hence, by the quotient rule, $\partial E(q \mid g) / \partial \alpha$ is positive if and only if

$$
\int_{\underline{q}}^{\bar{q}} q R_{\alpha}(q ; \alpha) d F(q) \int_{\underline{q}}^{\bar{q}} R(q ; \alpha) d F(q)-\int_{\underline{q}}^{\bar{q}} R_{\alpha}(q ; \alpha) d F(q) \int_{\underline{q}}^{\bar{q}} q R(q ; \alpha) d F(q)>0 .
$$

Using the linear form for $R$ in (6), we have that $R_{\alpha}=b+q$. Expanding and simplifying (15), the expression reduces to

$$
a \operatorname{Var}(q)>0
$$

which always holds by the assumption that $a>0$. Hence $E(q \mid g)$ increases in $\alpha$. By a similar analysis, $E(q \mid b)$ decreases in $\alpha$, which implies that $\Delta$ increases in $\alpha$.

Proof of Proposition 3. Clearly no type of either firm will deviate to $p<p^{*}(q) \cdot p^{*}(\bar{q})<\bar{q}$ cannot hold under D1 criterion for the same reason as in Proposition 1. Namely, for a price $p^{\prime}$ just above $p^{*}(\bar{q})$, the only types for which such deviation is profitable for the largest set of best responses are close to $\bar{q}$, but then consumers would purchase at $p^{\prime}$, and therefore $\bar{q}$ type would deviate to $p^{\prime}$ because, at worst, belief will be the same for $\bar{q}$ (so second period profits will not fall) and profit will be higher in the first period because $\bar{q}$ only sells to loyals and will continue to do so but at a higher price. We thus must have $p^{*}(\bar{q})=\bar{q}$.

For equilibrium existence we need to show that each firm's profit function is concave in $q^{e}$, and that $v(q)=q-p^{d}(q)$ is decreasing in $q$.

Let us take SOC for (11) and using $p^{*}(q)$ from (12) write

$$
\beta \Delta n\left(r_{q^{e} q^{e}}\left(q, q^{e}\right)-r_{q^{e} q^{e}}\left(q^{e}, q^{e}\right)-r_{q q^{e}}\left(q^{e}, q^{e}\right)\right)<0
$$

which is the same condition as in the monopoly case and is guaranteed by Assumption 1 parts (iv) and (v).

Given that profit functions are concave for all $q$, and any quality type can ensure positive profit by charging $p^{*}(\bar{q})$ in the first period, it has to be that profits are positive for all types. 
We now derive a sufficient condition for $v(q)=q-p^{*}(q)$ to be decreasing in $q$. This holds if we have $\frac{\partial p^{*}(q)}{\partial q}>1$ for all $q$. Upon taking derivative of $p^{*}(q)$ and some rearrangement, we obtain

$$
1<\frac{\beta \Delta n\left(f(q) \int_{q}^{\bar{q}} r_{q^{e}}(x, x) d x-r_{q^{e}}(q, q)(1+\lambda-F(q))\right)}{(1+\lambda-F(q))^{2}}+\frac{\lambda \bar{q} f(q)}{(1+\lambda-F(q))^{2}} .
$$

Using the assumption that $r_{q^{e}}(q, q)$ is negative and increasing in $q$, the above is implied by

$$
1<\frac{\beta \Delta n r_{q^{e}}(q, q)(f(q)(\bar{q}-q)-(1+\lambda-F(q)))}{(1+\lambda-F(q))^{2}}+\frac{\lambda \bar{q} f(q)}{(1+\lambda-F(q))^{2}} .
$$

Using our earlier assumption that $f(q)(\bar{q}-q)-(1-F(q)) \leq 0$ we can see that the first term is positive, so for $p^{\prime}(q)>1$ it is sufficient to require

$$
n \geq \max _{q \in[\underline{q}, \bar{q}]} \frac{(1+\lambda-F(q))^{2}-\lambda \bar{q} f(q)}{\beta \Delta r_{q^{e}}(q, q)((\bar{q}-q) f(q)-(1+\lambda-F(q)))} .
$$

Note that when $\lambda \bar{q} f(q)<(1+\lambda-F(q))^{2}$ holds for all $q$ we have $\underline{n}^{d}<0$, so that the condition on $n$ is not binding.

\section{A model of endogenous review creation with consumer effort}

Throughout the paper, we have assumed existence of a probabilistic review function $r\left(q, q^{e}\right)$ with $r_{q}>0$ and $r_{q^{e}}<0$. This apparently reflects the way consumers write reviews very well. In this section, we will give another possible justification about why consumers might create reviews according to a reduced-form function $r\left(q, q^{e}\right)$.

Instead of imposing an exogenous review function $r$, we now consider a situation in which consumers have to exercise costly effort in order to make a product operate properly. Consumer choose effort, depending on expected product quality. If a consumer expects that the iPhone she purchases is of high quality, she expects that it just works without much preparation or adjustments, and is more likely to blame the product for not working properly. A better chair should be usable on the spot without requiring substantial customization.

The probability of a success is now given by a success function $s(q, e)$, which increases both in true product quality $q$ and in effort $e$. Early period consumers report whether using the product was a success or not, which serves as a signal of quality for late period consumers. 
As we will show, a review function that is used throughout the paper can be derived from the success function $s(q, e)$ and cost of effort $c(e)$.

More formally, as before, a monopolist firm privately observes quality $q$, drawn from a twice continuously differentiable distribution $F(q)$ with support $[\underline{q}, \bar{q}]$. Marginal cost is assumed to be constant, independent of the quality and normalized to zero. There is a mass 1 of identical consumers in period 1, whose review simply states whether using the product was a success or not. In period 2 , a mass $n>0$ of consumers update their beliefs about product quality depending on the signal from period 1 consumers. Crucially, period 2 consumers do not observe neither expected quality nor effort of period 1 consumers.

The utility derived from using a product depends on the expected quality $q^{e}$ and effort $e \in[0,1]:$

$$
u\left(q^{e}, e, p\right)=v s\left(q^{e}, e\right)-c(e)-p
$$

where $v$ is the valuation in case of success, which will be normalized to $v=1$ in the following, and $p$ is the price chosen by the firm.

Still denoting partial derivatives with subscripts as before, we assume that $s_{q}>0$, $s_{e}>0$ (probability of success increases both in quality and effort) and $s_{e q}<0$ (higher effort pays off less at higher quality levels), $s_{e e} \leq 0$, and $c_{e}>0$ and $c_{e e}>0$. All higher order derivatives are assumed to be 0 .

Therefore, equilibrium effort level $e^{*}\left(q^{e}\right)$ is given by the solution to

$$
s_{e}\left(q^{e}, e^{*}\right)=c_{e}\left(e^{*}\right)
$$

where our assumptions on $s$ and $c$ imply that $e^{*}\left(q^{e}\right)$ is decreasing in $q^{e}$.

Consumers anticipate that they will exert $e^{*}$ upon purchase, and hence there willingness to pay for a product of expected quality $q^{e}$ is

$$
\bar{u}^{*}\left(q^{e}\right)=s\left(q^{e}, e^{*}\left(q^{e}\right)\right)-c\left(e^{*}\left(q^{e}\right)\right)
$$

where our assumptions imply that $\bar{u}^{*}\left(q^{e}\right)$ is increasing in $q^{e}$. If usage was a success (which happens with probability $s$ ), a good review is created, and a bad review otherwise.

Proceeding with backwards inductions in an analogous manner as in Section 2, all quality types will charge the same price conditional on the good or bad review. Expected quality after review $k \in\{g, b\}$ is calculated with

$$
E(q \mid k)=\int_{\underline{q}}^{\bar{q}} p(q \mid k) q d q
$$


where the conditional density $h(q \mid k)$ is calculated with Bayes' rule, i.e.,

$$
\begin{aligned}
& h(q \mid g)=\frac{s\left(q, e^{*}(q)\right) f(q)}{\int_{\underline{q}}^{\bar{q}} s\left(x, e^{*}(x)\right) f(x) d x} \\
& h(q \mid b)=\frac{\left(1-s\left(q, e^{*}(q)\right)\right) f(q)}{\int_{\underline{q}}^{\bar{q}}\left(1-s\left(x, e^{*}(x)\right)\right) f(x) d x} .
\end{aligned}
$$

Second period prices conditional on review $k$ are thus equal to $p_{2}^{k}=\bar{u}(E(q \mid k))$, and we define $\Delta=p_{2}^{g}-p_{2}^{b}>0$.

Thus, as before we have that in a separating equilibrium, profits are given by

$$
\pi\left(q^{e}\right)=p\left(q^{e}\right)+n\left(p_{2}^{b}+s\left(q, e^{*}\left(q^{e}\right)\right) \Delta\right)
$$

with the remainder of the analysis proceeding as in Section 2.

Note that in order to have a separating equilibrium, we require that $s\left(q, e^{*}\left(q^{e}\right)\right)$ increases in $q$, decreases in $q^{e}$, and a positive cross-derivative. All three requirements are implied by our assumptions on $s$ and $c$.

Finally, since $e^{*}$ is determined in equilibrium, we can define a function

$$
r\left(q, q^{e}\right)=s\left(q, e^{*}\left(q^{e}\right)\right)
$$

which satisfies all properties we required throughout. This establishes qualitative equivalence of the two specifications. 